\title{
Conceptos teóricos de la evaluación de las estrategias de comunicación en social media
}

\author{
Emilia SMOLAK-LOZANO \\ Universidad de Málaga \\ emilia.smolak@gmail.com
}

Recibido: $12 / 11 / 2012$

Aceptado: 23/01/2013

\begin{abstract}
Resumen
En aras del reciente surgimiento de la importancia de elaborar unas bases teóricas y metodológicas de las investigaciones de comunicación 2.0 en el marco amplio de la investigación en comunicación, el presente trabajo pretende reconstruir y resumir las bases teóricas de la evaluación de comunicación realizada en Social Media como uno de los niveles más importantes y claves para la planificación estratégica de comunicación según el modelo RACE aplicándolo al marco de la investigación de Social Media. La metodología se basa en la revisión sistemática (sistematical review), incluyendo los métodos de estudio de escritorio y meta-análisis, lo que permite en este caso recopilar las fuentes y los análisis del concepto de evaluación en comunicación y adaptarlo a la nueva realidad 2.0. El fruto de la revisión histórico-teórica son varios modelos de evaluación con sus correspondientes descripciones metodológicas para ser aplicados a las investigaciones en Social Media.
\end{abstract}

Palabras clave: investigación, comunicación 2.0, Social Media, evaluación, planificación estratégica.

\section{Theoretical Concepts of the Evaluation of the Communication Strategies in Social Media}

\begin{abstract}
With the background of growing importance to elaborate the theoretical and methodological basis for communication 2.0 investigations within wider framework of communication investigations in general, the present study aims to reconstruct and resume the theoretical concepts de evaluation of communication in Social Media. As evaluation of communication is the top and key level in the strategic planning of communication according to RACE model, it is the attempt to adjust it to Social Media research reality. The Desk Research is the main method here in order to gather the sources and analysis of the evaluation concepts in communication in order to be reconstructed and applied, according to the requirements of 2.0 realities. The result of the historical and theoretical analysis is the list of the possible evaluation models with its corresponding methodological descriptions to be applied to the researches on Social Media.
\end{abstract}

Keywords: investigations, communication 2.0, Social Media, evaluation, strategic planning.

\section{Referencia normalizada}

SMOLAK-LOZANO, Emilia (2013): "Conceptos teóricos de la evaluación de las estrategias de comunicación en social media”. Estudios sobre el Mensaje Periodístico. Vol. 19, Núm. especial marzo, págs.: 469-479. Madrid, Servicio de Publicaciones de la Universidad Complutense.

Sumario: 1. Introducción. 2. Metodología. 3. La base teórica y los modelos clásicos de evaluación; 3.1. El concepto y definición de la evaluación en comunicación; 3.2. Síntesis de los modelos clásicos de evaluación. 4. Conclusiones. 5. Referencias bibliográficas.

\section{Introducción}

Las estrategias 2.0 predominan actualmente en las actividades de comunicación organizacional debido a su bajo coste de implementación, enfoque en el público y po- 
sibilidad de medición, lo que concuerda con los últimos paradigmas de comunicación online interactiva (Sheldrake, 2011). Sin embargo, se despierta bastante incertidumbre en cuanto a la evaluación de su eficacia y efectividad entre los profesionales, los negocios que las desarrollan y los científicos. A la vista de la observada digitalización de comunicación se han cambiado los modelos de evaluación, que necesitan un enfoque integral y holístico para aplicar los modelos y soluciones adecuadas como respuesta a las confusiones terminológicas acerca de cómo evaluar las estrategias de comunicación en el entorno de Social Media.

En 2011 se ha publicado el libro de Nobell Álvarez sobre la evaluación en comunicación como parte de la planificación estratégica siendo uno de los pocos e importantes escritos sobre el tema. En él se incluyen definiciones, rol y posición de la evaluación dentro del modelo RACE. También intenta resumir todas las publicaciones hasta la fecha sobre los modelos que se pueden emplear en el proceso de evaluación de las estrategias de comunicación y los elementos del proceso de planificación estratégica. Como tal, constituye la principal fuente y ha servido como inspiración de este articulo debido a su desarrollo comprehensivo. Debido a la escasez de publicaciones académicas sobre la evaluación de la comunicación en el mundo 2.0, el predominio de las publicaciones de proveniencia profesional y sobre todo enfocadas en Social Media Marketing, se necesita un análisis inicial de la base metodológica y teórica de la evaluación de comunicación en el entorno 2.0 y es de alta importancia abordar el tema del concepto y modelos de la evaluación aplicados a Social Media, desde el punto de vista académico de las ciencias de comunicación y la metodología de investigación social. Por lo cual, respondiendo a las necesidades del entorno comunicativo actual, este estudio pretende formar un punto de partida para futuros análisis sobre la evaluación de la comunicación 2.0 , en cuanto a su definición, estructura del proceso y modelos de aplicación a las redes sociales.

\section{Metodología}

Este estudio utiliza la metodología de la revisión sistemática- systematic review (Petticrew y Roberts, 2006; Cooper y Hedges, 1994; y Cooper, 1998) y meta-análisis narrativa (Greenhalgh, et al., 2009: web) y utiliza las técnicas de análisis de datos e informaciones secundarias (Churchill, 2002: 250). En el estudio se ha recopilado e investigado las publicaciones relacionadas con el tema publicadas a lo largo del desarrollo de las ciencias de la comunicación, con la intención de responder a la pregunta que guía esta investigación: ¿cómo se conceptualiza, define y diseña la evaluación y su proceso dentro de la investigación de comunicación enfocándola a la realidad 2.0?

\section{La base teórica y los modelos clásicos de evaluación}

Para empezar hay que destacar:

"Diversos autores utilizan indistintamente los conceptos «investigación»y «evaluación» para referirse a 'la aplicación sistemática de procedimientos de investigación de las ciencias sociales para evaluar la conceptualización, diseño, implantación y utilidad de los programas de intervención social'” (Cuttlip y Center, 2001, en: Nobell Álvarez, 2011: 78). 
Sin embargo, el concepto de evaluación necesita una definición más precisa para que no se confunda con el proceso de investigación per se, sino que señala directamente su papel dentro de la investigación de comunicación y planificación estratégica de comunicación en general.

\subsection{El concepto y definición de la evaluación en comunicación}

La RAE define evaluación como "señalar el valor de algo", lo que ya indica que el objetivo de este proceso de investigación es la estimación del valor de una actividad realizada. Al trasladarse al campo de la comunicación, Nobell Álvarez (2011: 78) nos aporta la siguiente definición:

"Tomando como base el modelo RACE, Wilcox (2001) define a la Evaluación: "como el paso dentro del proceso de relaciones públicas que consiste en a medición de los resultados respecto de los objetivos definidos durante el proceso de planificación" (Nobell Álvarez 2011: 203).

Para James Bissland este proceso consiste en "la valoración sistemática de un programa y sus resultados. La evaluación es la última etapa del plan estratégico de comunicación según el modelo RACE: Research, Planning, Communication, y Evaluation de Marston (1963) lo cual también destaca la necesidad de investigación previa antes de crear e implementar las estrategias comunicativas (también importante en Social Media):

"Esta visión que integra a la Evaluación a todo el proceso de planificación estratégica de la comunicación, es la misma que sostiene Margarida Maria Krohling Kunsch (2003, pág. 53) diciendo que la evaluación "tiene un función de carácter permanente, iniciándose en el planeamiento y desenvolviéndose en el acompañamiento de las acciones en ejecución, con propuestas para las correcciones necesarias y procedimientos para verificar los resultados deseados" (Nobell Álvarez, 2011: 78).

En cuanto a los métodos de investigación de los programas comunicativos de masas, Dominick y Wimmer, (2008: 559-581), se refieren sobre todo a las Relaciones Públicas y sus métodos de investigación, por lo que podemos considerar diversos tipos de evaluación: auditoría, análisis de los resultados del programa concreto, evaluación de la efectividad y desarrollo de un análisis basado en métodos cualitativos.

En el caso de la comunicación en Social Media, la evaluación es una función también económica que nos permite identificar la relación entre coste y beneficio, por lo cual necesitamos parámetros adecuados para la medición, es decir, criterios de evaluación y herramientas de evaluación (Hainsworth y Wilson, 1992: 11, en: Nobell Álvarez, 2011: 53 y 57).

A los dos tipos de evaluación reconocidos por Nobell Álvarez (2011:51-52): sumativa (evaluación del programa antes de su implementación en cuanto a sus objetivos fijados) y formativa (diagnóstico de la mejor estrategia a implementar dentro de un programa), se debe añadir el seguimiento de un programa o plan de comunicación que se realiza durante el programa (monitorización) y al acabar el programa, es decir, una investigación evaluativa relacionada con el programa especifico de la intervención comunicativa con el fin de estimar el grado de influencia de las actividades realiza- 
das y sus efectos, lo que puede implicar el uso de varios métodos de investigación (triangulación), siempre de acuerdo con el carácter de la variable de respuesta (response variable), tal como lo describe Babbie (2001:369-394).

Solamente entonces se puede obtener una imagen completa de las actividades de comunicación antes, durante y después de su implementación. Esto es especialmente válido cuando tenemos que contar con la realidad 2.0, que es un entorno susceptible a los cambios, que funciona en tiempo real y demanda constante control de los resultados para poder ajustar el comportamiento comunicativo empresarial a los nuevos datos y hechos.

En la planificación estratégica de comunicación, la evaluación forma parte clave como un elemento del proceso integral y global. Similarmente, se tiene que planificar todo el proceso de evaluación, que podemos aplicar a la comunicación 2.0, tal como ha sido desarrollado por Nobell Álvarez (2011: 101-102), señalando la importancia de que los indicadores y las herramientas estén en conformidad con el modelo de gestión organizacional y los objetivos corporativos, de definir los objetivos específicos de comunicación relacionadas con las metas organizacionales, de la conceptualización de las variables claves, de determinar las dimensiones e indicadores de forma clara y precisa que reduzca los niveles de abstracción, de identificar las herramientas adecuadas de medición, de diseñar las variables y evaluación que permita obtener el seguimiento del posible cambio de valores, de elaborar índices y por último, de la creación, elaboración y presentación de los informes.

Este procedimiento se demuestra de la mejor manera a través de la herramienta del modelo. Aunque se le considera una ilustración simplificada (Berganza Conde, et al., 2005: 25-27), puede resultar útil y adecuado para aplicar como una presentación de los pasos a seguir en la planificación de la evaluación.

\subsection{Síntesis de los modelos clásicos de evaluación}

A la hora de planificar cualquier tipo de investigación en Social Media y dentro del entorno 2.0, cuyo objetivo es la valoración de la estrategia completa y las políticas comunicativas, el uso de los modelos con el fin de programar una evaluación adecuada es imprescindible en Social Media. A lo largo del desarrollo de la ciencia de la comunicación se han creado múltiples modelos de evaluación de orígenes tanto académicos como profesionales. Dependiendo del objetivo de la evaluación y su dimensión, podemos elegir entre diversos modelos.

La identificación de las dimensiones de evaluación y el modelo más adecuado, que mide y valora la dimensión u objetivo particular, nos va a permitir una evaluación correcta, coherente y eficaz, con el fin de la implementación en la investigación del entorno comunicativo de Social Media.

El autor (Nobell Álvarez, 2011: 57-68) ha destacado y listado de forma completa los siguientes tipos de evaluación y sus correspondientes modelos. Hemos seleccionado los que pueden ser aplicados a una investigación de comunicación $2.0^{1}$ :

1 Todos los ejemplos de modelos presentados en las siguientes páginas provienen de Nobell (2011: 52-71). 
1) Evaluación de resultados, tal como lo describen Wimmer y Dominick (2008: 559-580). Al finalizar el proceso de comunicación se comprueba si se ha cumplimentado los objetivos y se evalúa el valor aportado. Hemos elegido dos ejemplos en esta categoría de los modelos de evaluación de resultados que ilustran los dos tipos de acciones más comunes que se realizan en Social Media:

- Estrategias de visibilidad e influencia en plataformas sociales a largo plazo (modelo clásico de Piedra Rodríguez, 2002 de evaluación de todo el proceso)

- Campañas virales y promocionales de la marca a corto plazo (los resultados directos de una acción realizada en base de un diagnostico previo de una situación que una empresa quiere cambiar, modelo de Andrade, 2005).

2) Evaluación correctiva y de resultados. Podemos usarla para el seguimiento de las estrategias y campañas en Social Media ya que aportan por un lado evaluación continua y carácter retroalimentatorio que permite modificar las acciones comunicativas e implementarlas de nuevo y evaluar los resultados obtenidos. "Estos modelos incorporan el concepto de control de gestión" (Nobell Álvarez, 2011:62). Por su carácter estratégico y validez en procesos de control de gestión, asimismo debido al elemento de retroalimentación, son modelos muy importantes para evaluar comunicación 2.0. Se pueden aplicar para el seguimiento y monitorización de la marca digital así como reputación o para monitorizar el rendimiento de la estrategia realizada en los canales de Social Media, ya que su carácter continuo y correctivo de resultados en tiempo real permite introducir cambios adecuados si son necesarios. De hecho, hemos divido esta categoría en consiguientes 4 sub-categorías de los modelos según el objetivo de aplicación. Cada uno de los cuatro tipos de modelos se encuentra en aplicación diaria en comunicación 2.0, donde la valoración del plan comunicativo, reputación, gestión de marca online y control de gestión de comunicación son los elementos claves del éxito en este entorno:

2a) Modelos de monitorización del plan comunicativo ejecutado (Ehling, 1985; Libaert, 2000) demuestran el papel de acción prosecutiva, monitorización constante, necesidad de planificar la posibilidad de ajustes, medición de resultados y rendimiento.

2b) Modelos de seguimiento de la reputación (Lesly, 1971 y 1980).

2c) Seguimiento de visibilidad y gestión de marca digital (Canillli, 1993; Martín Martín, 1998).

Estas dos categorías de los modelos se basan en la importancia de investigar el entorno y ambiente general acerca de la marca, valoración de los resultados, retroacción y ajuste en función del cambio y acción del entorno y sobre todo investigación de opinión del público e identificación de los públicos estratégicos desde el punto de vista de la empresa.

2d) Modelos que permiten control de gestión de proceso comunicativo (Illescas, 2000; Garrido, 2001), destacan el rol de dirección y gestión en plan comunicativo y el control sobre la gestión de comunicación en una entidad organizativa, de tal modo que se convierten en herramientas de gran importancia en el caso de Social Media, donde la gestión y control de comunicación deberían ser puntos claves y obligatorios para cualquier Dircom. 
3) Modelos circulares de evaluación:

"Este grupo contempla las etapas anteriores de control de resultado y seguimiento del proceso, pero además prevén un modelo circular en el que los resultados se vuelven un input (a modo de feedback) para la etapa inicial son modelos sistémicos, por lo tanto de comportamiento cíclico" (Nobell Álvarez, 2011: 62-64).

Los modelos tales como Grunig (et al., 1992), Xifra y Fita (1996) y de Xifra (1999), por su carácter general y circular, nos pueden servir en la aplicación de la evaluación holística de todo el proceso de comunicación corporativa, tanto offline como en Social Media. En este caso podemos valorar y controlar el input contribuido por Social Media al efecto en otros canales comunicativos, seguir este entorno 2.0 y monitorizar el $f e$ edback de los públicos destinatarios de comunicación corporativa. Además, se destaca aquí el carácter estratégico del input evaluado y valorado para la toma de decisiones estratégicas por parte de la junta directiva de la empresa, que pueden influir en determinar metas y objetivos estratégicos y la implementación futura o posibles cambios de estrategia corporativa de comunicación en todos los canales seleccionados.

La evaluación del posible éxito de la actividad comunicativa y de cambio (o no) efectuado en el entorno de la organización forma parte del proceso de decisión, así como el seguimiento de efectos causados y programas implementados, ya que Social Media se convierte en elemento clave de la comunicación estratégica, causando la necesidad de poder evaluar su input exacto en las estrategias generales de comunicación en una organización. Además, el seguimiento continuo de input y su medición y valoración al instante permite cambiar a las demás estrategias en otros canales, lo que es especialmente importante debido a la rapidez e inmediatez de los efectos de Social Media.

4) Medición de impacto. Definido en términos de imagen y cambio de actitud o de efectuar un comportamiento deseado dentro del público de una plataforma social online (partiendo de Babbie, 2003: 269). "Son modelos complejos, y en muchos casos conllevan la comparación entre los atributos iniciales (Identidad) y los atributos percibidos en determinado público (imagen)" (Nobell Álvarez, 2011: 66). En este caso los aplicamos en la investigación de la publicity y reputación de marca social online, valoración de la influencia o grado y tipos de involucración de los usuarios de redes sociales en acciones de la marca, tal como señala el modelo de comunicación por objetivos de Andrade (2005).

Además, estos modelos destacan la medición, valoración y control de efecto logrado para poder saber si la acción realizada sobre los públicos ha conseguido los cambios deseados. Incluye un punto clave acerca del diagnóstico mediante análisis DAFO de la identidad corporativa e imagen que podemos encontrar en el modelo de Capriotti (2004), lo que gana todavía más peso a la hora de programar y proyectar la marca digital en las redes sociales y ganarlas. Por último, nos enseñan también la importancia de la selección de objetivos comunicativos y sus correspondientes mensajes.

5) Retorno sobre la inversión.

Tal y como señala el autor de la exhaustiva lista de los diferentes tipos de modelos evaluativos en comunicación: 
"El modelo de Aenaldi (1968) es el único encontrado que hace una referencia directa a una medición de resultados de comunicación en términos de rentabilidad económica. Disciplinas como la publicidad y e marketing comprendieron mucho antes que la comunicación y las relaciones públicas la importancia de medir el ROI" (Nobell Álvarez, 2011:67).

El modelo de Arnaldi (1968), basándose en el trabajo de Nobell Álvarez (2011), lo presentamos como marco o punto de partida para las consiguientes consideraciones acerca de ROI en Social Media en los futuros análisis, ya que es un tema que provoca controversias y confusiones, por lo cual tiene que ser tratado aparte.

Retorno de Inversión (ROI) en Social Media toma diferentes formas y definiciones, dependiendo de los objetivos de comunicación, plataforma social y métricas correspondientes a estas (Powell, et al., 2011). Sin embargo, tal como señala este modelo, forma parte estratégica en el control ejecutivo de la gestión de comunicación, afectando el rendimiento de la empresa en su totalidad.

6) Modelos de la presencia mediática y producción. Utilizados en Social Media para evaluar la difusión del contenido y visibilidad de la marca/empresa en el entorno 2.0 .

"Este conjunto de modelos, que Wilcox (2000) clasificará de evaluaciones de tipo básicas, miden la "productividad" en comunicación y las repercusiones mediáticas que dicha producción genera." (Nobell Álvarez, 2011: 67).

En este grupo de modelos tales como García Falcón (1987) y Lester Potter (2004), al adaptar a estos modelos al mundo de Social Media, destacamos el papel de métodos tanto cuantitativos (alcance de difusión y grado de visibilidad) como cualitativos (tipos de soportes y producciones características para las plataformas sociales como por ejemplo social media release o enlaces). Además, se incluye la fase de producción de material comunicativo audiovisual, que en el caso de Social Media implica programación de las aplicaciones sociales y diseño gráfico, además de seguimiento de la difusión de este contenido a través de los canales de Social Media empleados.

7) Modelos interpretativos. "Son modelos que evalúan comportamientos y actitudes desde una perspectiva del "entendimiento". Emplean mayoritariamente técnicas cualitativas" (Nobell Álvarez, 2011: 68). Ambos modelos se enfocan en evaluar y valorar la actitud del público, grado de entendimiento mutuo entre el público y la empresa, valoración de los sentimientos del público hacia la empresa y su actividad en los canales de Social Media y por último, puede servir tanto como modelo de valoración final después de la implementación de un programa particular como para hacer un diagnóstico del entorno 2.0 de la empresa.

\section{Conclusiones}

Partiendo del trabajo de Nobell Álvarez (2011) y la clasificación elaborada en el apartado anterior, hemos intentado resumir la clasificación anteriormente analizada, desde el punto de vista de la aplicación práctica de estos modelos de evaluación al mundo de Social Media, presentándose su fruto en las siguientes tablas: 
Tabla 1

\begin{tabular}{|c|c|c|c|c|}
\hline \multicolumn{2}{|c|}{ Tipo del modelo } & Aplicación/objetivo & $\begin{array}{c}\text { Dimensión de la } \\
\text { evaluación en social } \\
\text { media }\end{array}$ & Métrica aplicada \\
\hline \multicolumn{2}{|c|}{ Evaluación de resultados } & $\begin{array}{l}\text { Valoración } \\
\text { resultado final }\end{array}$ & $\begin{array}{l}\text { Resumen de la } \\
\text { estrategia/campaña } \\
\text { social media }\end{array}$ & $\begin{array}{l}\text { Indicadores de rendimiento: } \\
\text { crecimiento de fans, aumento del } \\
\text { reconocimiento de la marca, } \\
\text { crecimiento en ventas, etc. }\end{array}$ \\
\hline \multirow{4}{*}{$\begin{array}{l}\text { Correctiva y } \\
\text { de resultados }\end{array}$} & $\begin{array}{l}\text { Monitorización } \\
\text { del plan de } \\
\text { comunicación/ } \\
\text { diagnóstico }\end{array}$ & $\begin{array}{l}\text { Monitorización } \\
\text { ajustar el plan }\end{array}$ & \multirow{4}{*}{$\begin{array}{l}\text { Control y monitorización } \\
\text { de los programas y } \\
\text { acciones en social media, } \\
\text { seguimiento de } \\
\text { conversaciones online, } \\
\text { determinar la marca } \\
\text { digital y reputación en } \\
\text { plataformas sociales, } \\
\text { gestión efectiva de social } \\
\text { media y comunidades } \\
\text { virtuales, presencia en las } \\
\text { plataformas sociales, } \\
\text { mejorar el reconocimiento } \\
\text { e imagen, rendimiento de } \\
\text { las estrategias social } \\
\text { media, posicionamiento } \\
\text { frente a la competencia }\end{array}$} & \multirow{4}{*}{$\begin{array}{l}\text { Cualitativos y cuantitativos: } \\
\text { - palabras clave } \\
\text { - alcance } \\
\text { - impresiones } \\
\text { - efectos de Social Media } \\
\text { - fans y "Me gusta" } \\
\text { - cantidad de conversaciones y } \\
\text { comentarios } \\
\text { - rasgos de marca percibidos } \\
\text { - actitudes dominantes } \\
\text { - canales -cuáles y cuántos- de } \\
\text { presencia } \\
\text { - beneficio/coste de aplicación de } \\
\text { Social Media } \\
\text { - tamaño de comunidad } \\
\text { - actividades realizadas } \\
\text { - ranking en buscadores }\end{array}$} \\
\hline & $\begin{array}{l}\text { Seguimiento de } \\
\text { reputación }\end{array}$ & $\begin{array}{l}\text { Control sobre } \\
\text { reputación }\end{array}$ & & \\
\hline & $\begin{array}{l}\text { Seguimiento de } \\
\text { visibilidad y } \\
\text { gestión de marca } \\
\text { digital }\end{array}$ & $\begin{array}{lcr}\text { Control } & \text { sobre } & \text { el } \\
\text { aumento } & \text { de } & \text { la } \\
\text { visibilidad/ } & \text { publicity } \\
\text { en Social } & \text { Media y } \\
\text { evaluación } & & \text { de } \\
\text { efectividad de gestión } \\
\text { de identidad digital }\end{array}$ & & \\
\hline & $\begin{array}{l}\text { Evaluación de la } \\
\text { dirección del } \\
\text { proceso de } \\
\text { comunicación/ } \\
\text { diagnóstica }\end{array}$ & $\begin{array}{l}\text { Evaluación de gestión } \\
\text { de comunicación } \\
\text { corporativa }\end{array}$ & & \\
\hline
\end{tabular}

Tabla 2

\begin{tabular}{|c|c|c|c|}
\hline $\begin{array}{l}\text { Tipo del } \\
\text { modelo }\end{array}$ & Aplicación/objetivo & $\begin{array}{c}\text { Dimensión de la evaluación en } \\
\text { social media }\end{array}$ & Métrica aplicada \\
\hline $\begin{array}{l}\text { Modelos } \\
\text { circulares }\end{array}$ & $\begin{array}{l}\text { Input y corrección } \\
\text { de plan } \\
\text { (diagnóstico) }\end{array}$ & $\begin{array}{l}\text { Adaptación y modificación de } \\
\text { estrategias de social media. } \\
\text { Aprendizaje y escucha }\end{array}$ & $\begin{array}{l}\text { De cualquier tipo: supone un cambio } \\
\text { necesario y afecta a las acciones de forma } \\
\text { significativa }\end{array}$ \\
\hline $\begin{array}{l}\text { Medición de } \\
\text { impacto }\end{array}$ & $\begin{array}{l}\text { Cambio de imagen } \\
\text { o actitud } y / o \\
\text { comportamiento }\end{array}$ & $\begin{array}{l}\text { Imagen y refutación } 2.0 \text {, influencia, } \\
\text { autoridad, involucración de } \\
\text { audiencia, mejorar la situación de una } \\
\text { empresa después de una crisis, } \\
\text { RR.PP. } 2.0\end{array}$ & $\begin{array}{l}\text {-Tipo de liderazgo de una marca } \\
\text { - Popularidad } \\
\text {-Imagen antes y después } \\
\text { - Grado de influencia y poder } \\
\text { - Número de fans y seguidores } \\
\text { - Tipos de acciones de la comunidad } \\
\text { - Tipo de interacciones con la comunidad } \\
\text { - Tipo de interacciones de la comunidad con } \\
\text { el contenido } \\
\text { Rasgos diferenciadores de posicionamiento }\end{array}$ \\
\hline ROI & $\begin{array}{l}\text { Evaluación de } \\
\text { relación entre costes } \\
\text { y beneficio }\end{array}$ & $\begin{array}{l}\text { Recursos aplicados en social media } \\
\text { en personal, tecnologías y cantidad de } \\
\text { dinero involucrada vs. beneficios } \\
\text { reales para el funcionamiento de la } \\
\text { empresa y su imagen }\end{array}$ & $\begin{array}{l}\text { - Costes de comunidad vs. mejoras de } \\
\text { servicios } \\
\text { - Retorno de conversaciones } \\
\text { - Retorno de influencia / Relación / } \\
\text { Engagement }\end{array}$ \\
\hline
\end{tabular}

Tabla 3

\begin{tabular}{|c|c|c|c|}
\hline $\begin{array}{l}\text { Tipo del } \\
\text { modelo }\end{array}$ & Aplicación/objetivo & $\begin{array}{c}\text { Dimensión de la } \\
\text { evaluación en social } \\
\text { media }\end{array}$ & Métrica aplicada \\
\hline $\begin{array}{l}\text { Presencia } \\
\text { mediática y } \\
\text { producción }\end{array}$ & $\begin{array}{l}\text { Cantidad y calidad } \\
\text { de contenido }\end{array}$ & $\begin{array}{l}\text { Difusión de contenido y } \\
\text { formatos distribuidos en } \\
\text { las plataformas sociales }\end{array}$ & $\begin{array}{l}\text { - Métodos de difusión dominantes } \\
\text { - Leads } \\
\text { - Si ayuda o no a fomentar publicity } \\
\text { - Cantidad del contenido } \\
\text { - Tipo de formatos } \\
\text { - Actitud del público hacia el formato } \\
\text { - Calidad del contenido } \\
\text { - Escala según público } \\
\text { - Formas de interacción (horas) } \\
\text { - Reacciones del público a diferentes tipos de contenidos } \\
\text { - Redes de presencia de contenido } \\
\text {-Impacto del contenido }\end{array}$ \\
\hline
\end{tabular}




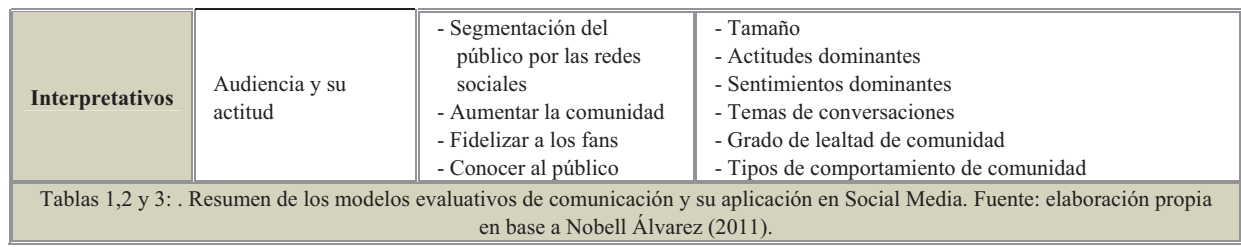

Como muestra la tabla, hemos elegido múltiples dimensiones de la evaluación y los tipos de investigación evaluativa, a los que corresponden a cada uno de los varios modelos anteriormente analizados. Asimismo, a la hora de diseñar el proceso de evaluación, primero se determinará la dimensión y tipo de evaluación lo que facilitará la selección del modelo adecuado y la planificación del proceso, así como durante su implementación y desarrollo del seguimiento, gestión y control por parte de Dircom. Teniendo en cuenta las necesidades de la comunicación 2.0, hemos destacado las siguientes dimensiones y sus modelos clásicos correspondientes, ya aplicados a este nuevo entorno en cuanto a los objetivos y métricas/indicadores:

- de la evaluación de los resultados de un programa de comunicación,

- correctiva y de los resultados ( tratados juntos por su carácter complementario) donde se encuentran la monitorización y seguimiento de la marca en el ecosistema de la red social, así como la monitorización de la reputación y visibilidad, control y gestión del proceso de la comunicación organizacional o actividad diagnostica de la estrategia,

- circular- retroalimentación de tal modo que se sigue la lógica de input-feedback-corrección,

- medición de impacto- donde se aporta y mide los indicadores del grado de la respuesta y del cambio (dimensión de efectividad en comunicación)

- de ROI- estimación de la relación entre coste y beneficio (dimensión de eficacia en comunicación),

- de producción mediática y del contenido audiovisual publicado en las redes para comprobar la reacción del público y facilitar la predicción de su potencial y posible respuesta del público,

- interpretativos- evaluación de la actitud de la audiencia hacia la marca.

Como podemos observar, la evaluación de comunicación 2.0 tiene que ser enfocada en los siguientes elementos (de forma integral o independiente): contenido, audiencia, eficacia: ROI y rendimiento, efectividad (resultados e impacto), monitorización de reputación e imagen, control de gestión, aplicación de mecanismos correctivos y de adaptación.

Entre los límites de este estudio encontramos la falta de modelos de evaluación formativa (diagnóstica), que es incluida dentro de los modelos circulares o correctivos, pero debería encontrarse también como apartado singular por su carácter específico y papel en la planificación. Este elemento también debería encontrarse entre las dimensiones claves de evaluación. Intentaremos tratar esto en próximas investigaciones. La segunda cuestión que podemos enmarcar como limitación del estudio resulta 
de su aplicación sobre todo a las Relaciones Públicas 2.0 y su modelo de planificación estratégica. Sin embargo, ya existen estudios que permiten justificar su aplicación en el marco más general de la comunicación organizacional 2.0 (por ejemplo, Comunicación Inbound).

Dada la diversidad de las dimensiones y métricas, que además pueden tener carácter transversal y dinámico, esta clasificación no se debería tratar de forma definitiva. Cada investigador de Social Media puede elegir tal modelo de evaluación que corresponde a sus objetivos comunicativos y empresariales, y a su vez a los determinados modos de medición. Los métodos, las técnicas y las herramientas de análisis de Social Media que resultan de la selección del modelo determinarán los indicadores de efectividad. Se recomienda siempre una construcción del modelo lo más comprehensivo, integral y global posible para que cubra la variedad de indicadores de la realidad 2.0.

Hay que tener presente, que este resumen tiene carácter orientativo, ya que Social Media debido a su dinamismo de desarrollo nos aporta alto grado de diversidad de estrategias y modos de evaluación. Es un ejemplo bien argumentado que las teorías clásicas y sus frutos son válidos en la nueva realidad comunicativa 2.0 y deberían ser utilizados o más bien re-utilizados, en su forma original o modificada y adaptada, en los análisis empíricos de Social Media, tanto en el mundo académico como en el profesional. La clasificación es la respuesta teórico-metodológica a la falta de fundamentos científicos en las investigaciones y evaluaciones de estrategias de comunicación en Social Media actuales. Puede servir como base inicial y punto de partida para crear los fundamentos metodológicos de las investigaciones de Social Media, sobre todo las enfocadas a la evaluación de estrategias de comunicación 2.0. La modelación adecuada de las diferentes dimensiones y tipos de evaluación ayuda a la planificación del proceso, así como la gestión del proceso de comunicación organizacional.

\section{Referencias bibliográficas}

BABBIE, Earl (2001): Badania spoteczne w praktyce. Varsovia, Wydawnictwo Naukowe PWN

BERGANZA CONDE, Ma Rosa; RUIZ SAN ROMÁN, José A. (ed., 2005): Investigar en Comunicación. Madrid, McGraw Hill

CHURCHILL, Gilbert A. (2002): Badania marketingowe. Podstawy metodologiczne. Varsovia, Wydawnictwo naukowe PWN

COOPER, Harris (1998): Synthesizing research: A guide for literature reviews (3rd ed.). California, Sage Publications

COOPER, Harris; HEDGES, Larry V., (ed., 1994): The Handbook of Research Synthesis. Nueva York, Russel Sage Foundation

GREENHALGH, Trisha; POTTS, Henry W.W.; WONG, Geoff; BARK, Pippa; SWINGLEHURST, Deborah (2009): "Tensions and Paradoxes in Electronic Patient Record Research: A Systematic Literature Review Using the Meta-narrative 
Method". Milbank Quarterly, vol. 87, nº 4, Wiley Blackwell, pp. 729-88: http://discovery.ucl.ac.uk/18821/1/18821.pdf [ fecha de consulta: 22 de Septiembre de 2012]

MARSTON John E. (1963): The Nature of Public Relations. New York, McGrawHill Book Company, Inc.

NOBELL ÁLVAREZ, Alejandro (2011): Medición y evaluación en comunicación. Málaga, Instituto de Investigaciones de Relaciones Públicas

PETTICREW Mark; ROBERTS Helen (2006): Systematic reviews in the social sciences. Reino Unido, Wiley Blackwell

POWELL, Guy R.; GROVES Steven; DIMOS Jerry (2011): Social Media ROI. Singapore, Wiley \& Sons

SHELDRAKE, Peter (2011): The Business of Influence: Reframing Marketing and $P R$ for the Digital Age. Reino Unido, J. Wiley and Sons

WIMMER Roger D.; DOMINICK Joseph R. (2008): Mass media. Metody badań.Cracovia, Wydawnictwo Uniwersytetu Jagiellońskiego

\section{Emilia SMOLAK-LOZANO}

Universidad de Málaga

Facultad de Ciencias de la Comunicación

Doctoranda en Gestión Estratégica de Comunicación 2.0 y Account Manager de Agencia

NewRebel ES

emilia.smolak@gmail.com 